\title{
CLOSURE PROPERTIES OF $C(X)$ IN ITS SECOND DUAL
}

\section{SAMUEL KAPLAN}

1. Let $X$ be a fixed compact Hausdorff space, $C$ the Banach lattice of continuous real functions on $X, L$ its dual, and $M$ its second dual. The closure of $C$ in $M$ under the weak topology $w(M, L)$ determined by $L$ is, as is well known, $M$ itself. In fact, the closure of $C$ under the Mackey topology $\tau(M, L)$ is $M$ itself, by the Grothendieck Theorem. A deeper property is that this relationship between $C$ and $M$ also holds under order-convergence, which is finer than $\tau(M, L)$-convergence. Let us examine this in more detail. An order-bounded net $\left\{f_{\alpha}\right\}$ in $M$ converges to $f \in M$ if $f=\lim \inf _{\alpha} f_{\alpha}=\lim \sup _{\alpha} f_{\alpha}$, where $\lim \inf _{\alpha} f_{\alpha}=\bigvee_{\alpha}\left(\bigwedge_{\beta>\alpha} f_{\beta}\right)$ and $\lim \sup _{\alpha} f_{\alpha}=\Lambda_{\alpha}\left(\bigvee_{\beta>\alpha} f_{\beta}\right)$. If a set $A$ contains all such limits of order-convergent nets of $A$, we say $A$ is closed under order-convergence, or simply closed. In general a set $A$ is not closed. However there exists a smallest closed set containing $A$, and it is this set which is called the closure of $A$. With this definition we have the property stated above: the closure of $C$ under order-convergence is $M$ itself.

Unlike the case of topological convergence, the closure of a set $A$ cannot in general be obtained by adjoining to $A$ all limits of orderconvergent nets of $A$. If we adjoin all such limits, the enlarged set need not be closed, and it may be necessary to iterate the process repeatedly, possibly a transfinite number of times, before every point of the closure is obtained. In particular, the set obtained by adjoining to $C$ all limits of order-convergent nets of $C$ is a proper subset of $M$. In [2] we gave this set the symbol $U$, since it corresponds to those bounded functions on $X$ which are integrable with respect to every Radon measure ("universally" integrable), and we have studied its properties in that and subsequent papers. Beyond $U$ we did not go, only conjecturing $[3, \S 4]$ that it would require an uncountable number of iterations of the above process to obtain all of $M$.

Contrary to the conjecture, we now present a proof that every element of $M$ is the limit of an order-convergent net of $U$. Thus all of $M$ can be obtained from $C$ by one iteration of the operation of adjoining limits of order-convergent nets.

2. The theorem we actually prove is considerably stronger than the above statement. We call a set $A \sigma$-closed, if it contains all limits of order-convergent sequences of $A$. The smallest $\sigma$-closed set con-

Received by the editors June 11, 1965. 
taining a set $A$ is called the $\sigma$-closure of $A$. Following function theory usage, we call the $\sigma$-closure of $C$ the Baire subspace of $M$, and denote it by $B a . U$ is $\sigma$-closed [2, (8.2)], hence we have $C \subset B a \subset U$. As with nets, we can iterate the process of adjoining limits of order-convergent sequences. The set obtained by adjoining such limits to $C$ is called the first Baire class and denoted by $B a^{1}$; the set obtained by doing this to $B a^{1}$ is called the second Baire class $B a^{2}$; and so forth. We can now state our theorem.

THEOREM 1. Every element of $M$ is the limit of an order-convergent net of $B a^{2}$.

Before proceeding with the proof, we recall some properties of $L$ and $M[2 ; 3]$. Given an element $\mu$ of $L$, we will call the closed (vectorlattice) ideal $I$ in $L$ generated by $\mu$ a principal closed ideal. In what follows $I$ 's will always denote principal closed ideals of $L$. Given $I_{1}, I_{2}$, then $I_{1}+I_{2}$ is also a principal closed ideal, and thus the family $\{I\}$ of all principal closed ideals of $L$ is a directed set under inclusion.

Each $I$ is a topological direct summand of $L$, hence its dual is a direct summand of $M$. We denote it by $M_{I}$, and for each $f \in M$, we denote the component of $f$ in $M_{I}$ by $f_{I}$. For use below, we note the trivial property that for any $\mu \in I, f_{I}(\mu)=f(\mu)$. (Incidentally, we use $f(\mu)$ and $\mu(f)$ interchangeably: $f(\mu)=\mu(f)$.)

$M_{I}$ is actually a closed ideal in $M$, hence the projection operation $f \rightarrow f_{I}$ of $M$ onto $M_{I}$ preserves suprema and infima:

(i) If $f=\mathrm{V}_{\alpha} f_{\alpha}$, then $f_{I}=\mathrm{V}_{\alpha}\left(f_{\alpha}\right)_{I}$; and similarly for $\Lambda$.

We will also need the well-known property (cf. the proof of (12.3) in $[2])$ :

(ii) For each $I$, the projection of $M$ onto $M_{I}$ maps $B a^{2}$ onto $M_{I}$.

(Since $I$ can be identified with $\mathfrak{L}^{1}(\mu), \mu$ any element generating $I$, then $M_{I}$ can be identified with $\mathscr{L}^{\infty}(\mu)$, so the above is simply the common statement that every element of $\mathscr{L}^{\infty}(\mu)$ has a representative in $B a^{2}$.)

We turn to the proof of the theorem. Consider $f \in M$, and we can confine ourselves to the case $0 \leqq f \leqq 1$. For each $I$, choose $g(I) \in B a^{2}$ such that $(g(I))_{I}=f_{I}$. Moreover, replacing $g(I)$ by $(0 \vee g(I)) \wedge 1$ if necessary, we can assume $0 \leqq g(I) \leqq 1$ (from (i), $[(0 \vee g(I)) \wedge 1]_{I}$ $\left.=\left(0 \vee(g(I))_{I}\right) \wedge \mathbf{1}_{I}=f_{I}\right)$. The collection $\{g(I) \mid$ all $I\}$ is a net, since the $I$ 's form a directed set. We show $\lim \sup _{I} g(I)=f$; as the same argument will hold for $\lim \inf _{I} g(I)=f$, this will establish the theorem.

Set $h=\lim \sup _{I} g(I)$. What we have to show is that $h(\mu)=f(\mu)$ for all $\mu \in L$. It is enough to show

(iii) $h_{I}=f_{I}$ for all $I$, because then, given any $\mu \in L$ and choosing for $I$ the principal closed 
ideal generated by $\mu$, we have $h(\mu)=h_{I}(\mu)=f_{I}(\mu)=f(\mu)$.

For each $I$, set $h(I)=\mathrm{V}_{J \supset I} g(J)$; then by definition, $h=\Lambda_{\mathrm{al1}} I h(I)$. Now $J \supset I$ implies $(g(J))_{I}=\left((g(J))_{J}\right)_{I}=\left(f_{J}\right)_{I}=f_{I}$. It follows from (i) not only that $(h(I))_{I}=f_{I}$, but that $(h(J))_{I}=f_{I}$ for every $J \supset I$. Applying (i) again, this time to $h=\Lambda_{\mathrm{all} I} h(I)$, we have $h_{I}=f_{I}$ for all $I$.

3. Let $E$ be a sub-vector-lattice of $M$ (we restrict ourselves to a sub-vector-lattice for convenience). There is a more refined process for obtaining the closure of $E$ than the iterated adjunction of limits of order-convergent nets. Let us denote by $D(E)$ the Dedekind closure of $E$ : the set of elements of $M$ each of which is both a supremum of some subset of $E$ and an infimum of some subset of $E . D(E)$ is also a sub-vector-lattice. It is Dedekind-closed, hence the operation cannot be iterated. A larger sub-vector-lattice is the linear subspace generated by the elements of $M$ each of which is a supremum of some subset of $E$ or an infimum of one. We denote it by $S(E)[2, \S 6]$. It is easily verified that $S(E)=S(D(E)$ ), so if we use the latter notation, we can write

$$
E \subset D(E) \subset S(D(E))
$$

This process can now be iterated, and we obtain an ascending chain:

$$
E \subset D(E) \subset S(D(E)) \subset D(S(D(E))) \subset \cdots .
$$

How is this chain related to the ascending chain obtained by adjunction of limits of order-convergent nets? It is easy to see that the set of limits of order-convergent nets of $E$ is contained in $D(S(D(E)))$. An immediate question is whether equality necessarily holds. If such is the case, then each even term in the above chain (from the fourth term on) is the set of limits of order-convergent nets of the preceding even term. We do not know whether such is the case in general; however, for the situation in which we are interested, $E=C$, equality does hold. We first note that $C=D(C)$, that is $C$ is Dedekind-closed; next that $S(D(C))=S(C)$ is our space $S$ of semicontinuous elements; finally, that $D(S(D(C)))$ is our space $U[2]$. The first six terms of the above chain thus become

$$
C=C \subset S \subset U \subset S(U) \subset D(S(U)) .
$$

That $U$ is the set of limits of order-convergent nets of $C$ was shown in $[2,(9.6)]$. That $D(S(U))$ is the set of limits of order-convergent nets of $U$ is trivial, since this latter set is all of $M$ (Theorem 1). Moreover, the chain has come to an end:

Theorem 2. $D(S(D(C)))=D(S(U))=M$. 
4. A glance at the chain in (4) raises an immediate question: what is the relation of $S(U)$ to $M$, and in particular, is it all of $M$ ? We present some properties of $S(U)$ in the direction of answering this.

We can write $L=L_{0} \oplus L_{1}$, where $L_{0}$ is the ideal of the atomic (Radon) measures and $L_{1}$ that of the diffuse, or purely nonatomic, ones. This gives the decomposition $M=M_{0} \oplus M_{1}$, and we will call $M_{0}$ the atomic part and $M_{1}$ the diffuse part of $M . M_{0}$ is isomorphic with the space of all bounded real functions on $X$. The first property of $S(U)$ which we note is that it contains $M_{0}$. To see this we remark that for each $x \in X$, the characteristic function of $x$ in $M_{0}$ is a u.s.c. element $[2,(6.8)]$, hence in $U$. Since every element of $\left(M_{0}\right)_{+}$is a supremum of finite linear combinations of such characteristic functions, $M_{0} \subset S(U)$.

A consequence of this is that the projection $(S(U))_{1}$ is identical with $(S(U)) \cap M_{1}$. For, given $f \in S(U), f-f_{1} \in M_{0} \subset S(U)$, hence $f_{1}=f+\left(f_{1}-f\right) \in S(U)$. Thus $S(U)=M_{0} \oplus\left[(S(U)) \cap M_{1}\right]$, and the problem is reduced to one on $M_{1}$.

We turn to another property of $S(U)$. In the remainder of the paper, given $\mu \in L$, we will denote the ideal $I$ generated by $\mu$ by $L_{\mu}$, and we will write $M_{\mu}, f_{\mu}$ for $M_{I}, f_{I}$.

THEOREM 3. For every $\mu \in L, M_{\mu} \subset S(U)$. More explicitly, each $f \in\left(M_{\mu}\right)_{+}$is an infimum of elements of $U$.

Proof. Consider a fixed $\mu \in L$, and since $L_{\mu}=L_{|\mu|}$, we can assume $\mu \geqq 0$.

Lemma 1. For every $\nu \in L, \nu>0$ such that $\mu \wedge \nu=0$, there exists a u.s.c. element $f$ satisfying:

$$
0 \leqq f \leqq 1, \quad \mu(f)=0, \quad \nu(f)>0 .
$$

For simplicity, assume $\nu(1)=1$. We will define by induction, a sequence $\left\{f_{n}\right\} \subset C$ such that

(ii) $\mu\left(f_{n}\right) \leqq 1 / 2^{n+1}$

$$
\begin{aligned}
& n=1,2, \cdots, \\
& n=1,2, \cdots .
\end{aligned}
$$$$
\text { (iii) } \nu\left(f_{n}\right) \geqq(1 / 2)+\left(1 / 2^{n+1}\right)
$$

Since $\mu \wedge \nu=0$, we can write $1=f_{1}+g_{1}, f_{1}, g_{1}>0$, such that $\mu\left(f_{1}\right) \leqq 1 / 4$, $\nu\left(g_{1}\right) \leqq 1 / 4$, whence $\nu\left(f_{1}\right) \geqq 3 / 4$ [1, Chapter II, §2, Proposition 3]. Assume $f_{1}, \cdots, f_{n-1}$ have been chosen to satisfy the above conditions. Then write $f_{n-1}=f_{n}+g_{n}, f_{n}, g_{n} \geqq 0$, such that $\mu\left(f_{n}\right) \leqq 1 / 2^{n+1}$, $\nu\left(g_{n}\right) \leqq 1 / 2^{n+1}$, whence $\nu\left(f_{n}\right) \geqq(1 / 2)+\left(1 / 2^{n}\right)-\left(1 / 2^{n+1}\right)=(1 / 2)$ $+\left(1 / 2^{n+1}\right)$. This establishes the induction and gives us our sequence. 
Now set $f=\Lambda_{n} f_{n}$. Then $\mu(f)=0$ and, since $\nu$ is continuous on $M$, $\nu(f) \geqq 1 / 2$.

Lemma 2. For every $\nu \in L, \nu>0$, such that $\mu \wedge \nu=0$, there exists $a$ component $e$ of 1 which is an element of $U$ and satisfies: $\mu(e)=0$, $\nu(e)>0$.

Let $f$ be the element obtained in Lemma 1 . Then $\bigvee_{n} f \wedge(n 1)$ is the desired $e$.

We now proceed to prove the theorem.

(a) The theorem is true for $\mathbf{1}_{\mu}$.

It is enough to show that $1-1_{\mu}$ is a supremum of elements of $U$. Let $\left\{\nu_{\alpha}\right\}$ be the set of elements $\nu>0$ of $L$ such that $\mu \wedge \nu=0$, and for each $\alpha$, denote the $e$ of Lemma 2 by $e_{\alpha}$. We show $1-1_{\mu}=V_{\alpha} e_{\alpha}$. Set $e=1-1_{\mu}-\bigvee_{\alpha} e_{\alpha}$ and suppose $e \neq 0$. Then there exists $\nu \in L, \nu>0$, such that $\nu(1-e)=0[3,(3.4)]$ (the $\Omega$ in this reference should read $\tilde{\Omega}$ ). On the one hand this says that $\nu\left(1_{\mu}\right)=0$, hence $\nu \wedge \mu=0$, and thus $\nu$ is a $\nu_{\alpha}$. On the other hand it says that $\nu\left(e_{\alpha}\right)=0$ for all $\alpha$. We thus have a contradiction, and (a) is established.

(b) The theorem is true for every component of $1_{\mu}$, and therefore for positive finite linear combinations of such components.

Every component is easily shown to be a $1_{\nu}$ for some $\nu \in L$, hence the argument of (a) applies. The rest of the statement is clear.

Now every element of $\left(M_{\mu}\right)_{+}$can be approximated normwise by positive finite linear combinations of components of $\mathbf{1}_{\boldsymbol{\mu}}$. Hence, to complete the proof, we need only show that the set of elements which are infima of elements of $U$ is closed under the norm. While easily proved directly, this is a corollary of

THEOREM 4. The set of elements which are infima of elements of $U$ is $\sigma$-closed.

Proof. Denote the set by $A$. It is easily verified that $A$ is closed under the operation of taking finite suprema. To prove the theorem, it will be sufficient to show $A$ is closed under countable suprema, that is, $\left\{f_{n}\right\} \subset A, f=\mathrm{V}_{n} f_{n}$ implies $f \in A$. And from our first observation, we can assume $f_{1} \leqq f_{2} \leqq \cdots$. Set $g=\Lambda\{h \in U \mid h \geqq f\}$; we show $g(\nu)$ $=f(\nu)$ for all $\nu \in L_{+}$, whence $g=f$.

Consider $\nu \in L_{+}$. Since $g \geqq f$, we need only show that for $\epsilon>0$, (i) $g(v) \leqq f(v)+\epsilon$.

We will obtain by induction a sequence $\left\{h_{n}\right\} \subset U$ satisfying:

(ii) $\quad h_{1} \leqq h_{2} \leqq \cdots$

(iii) $h_{n} \geqq f_{n}$

$n=1,2, \cdots$

(iv) $h_{n}(\nu) \leqq f_{n}(\nu)+\left(1-2^{-n}\right) \epsilon$

$n=1,2, \cdots$. 
Choose $h_{1} \in U$ such that $h_{1} \geqq f_{1}$ and $h_{1}(\nu) \leqq f_{1}(\nu)+\epsilon / 2$. Assume $h_{1}, \cdots$, $h_{n-1}$ have been chosen to satisfy (ii), (iii), (iv). Now $f_{n} \bigvee h_{n-1}=f_{n}$ $+\left(h_{n-1}-f_{n}\right)^{+} \leqq f_{n}+\left(h_{n-1}-f_{n-1}\right)$, hence $\left(f_{n} \bigvee h_{n-1}\right)(\nu) \leqq f_{n}(\nu)+h_{n-1}(\nu)$ $-f_{n-1}(\nu) \leqq f_{n}(\nu)+\left(1-2^{-(n-1)}\right) \epsilon$. Since $f_{n} \bigvee h_{n-1} \in A$, we can choose $h_{n} \in U$ such that $h_{n} \geqq f_{n} \vee h_{n-1}$ and $h_{n}(\nu) \leqq\left(f_{n} \bigvee h_{n-1}\right)(\nu)+1 / 2^{n} . h_{n}$ then satisfies (ii), (iii), (iv).

Since $\left\{f_{n}\right\}$ is bounded above, we can choose the $h_{n}$ 's so that $\left\{h_{n}\right\}$ is bounded above, and therefore $h=\mathrm{V}_{n} h_{n}$ exists. Since $U$ is $\sigma$-closed [2, (8.2)], $h \in U$; also $h \geqq f$; finally, $h(\nu)=\sup _{n} h_{n}(\nu) \leqq \sup _{n} f_{n}(\nu)+\epsilon$. It follows from the definition of $g$, that $g \leqq h$, hence (i) holds, and we are through.

Theorem 4 of course holds also for the set of elements which are suprema of elements of $U$. Whether $S(U)$ itself is $\sigma$-closed, or even norm-closed (not to mention equal to $M$ ), we do not know.

A FinAL REMARK. It is easy to show that $\sum_{\mu \in L} M_{\mu}=\cup_{\mu \in L} M_{\mu}$, is an ideal, is $\sigma$-closed, and is dense in $M$ (but is not equal to $M$ ). Thus, from Theorem 3, $S(U)$ contains a $\sigma$-closed ideal which is dense in $M$.

\section{BIBLIOGRAPHY}

1. N. Bourbaki, Integration, Chapters I-IV, Hermann, Paris, 1952.

2. S. Kaplan, On the second dual of the space of continuous functions, Trans. Amer. Math. Soc. 86 (1957), 70-90.

3. - The second dual of the space of continuous functions. II, Trans. Amer. Math. Soc. 93 (1959), 329-350.

Purdue University 